\title{
OPORTUNIDADES PERDIDAS EN MAGALLANES ${ }^{1}$
}

\section{SERGIO SOZA-AMIGO"Y PATRICIO AROCA GONZÁLEZ"}

\section{RESUMEN}

Muchos investigadores destacan la importancia de las relaciones entre sectores con el fin de sugerir medidas relativas al crecimiento económico (estudios sobre eslabonamientos o linkages), pero pocos, conjugan dicha información con los efectos que tiene los inputs primarios y la conmutación interregional en la economía. Aunque estos tipos de análisis son distintos, se encuentran estrechamente ligados cuando se pretende ahondar en razones que lleven a un crecimiento regional.

Este trabajo se concentra en el análisis de encadenamientos, salarios y conmutación regional, considerando distintos escenarios (economía abierta y cerrada). Los resultados obtenidos, dejan patente la evidente pérdida de recursos que se dejan de percibir en Magallanes, y que tiene como destino otras regiones, debido a la falta de políticas que apunten a la radicación de aquellas personas que más ganan para que vivan en la región.

PALABRAS CLAVES: Análisis Estructural Input-Output, Conmutación Interregional, Encadenamientos.

Clasificación JEL

A10, B41, C67, D57, O10, R15

Profesor Asociado, Facultad de Ciencias Económicas y Jurídicas, Universidad de Magallanes, Punta Arenas, Chile, e-mail: sergio.soza@umag.cl

* Profesor Titular, Departamento de Economía, Instituto de Economía Aplicada Regional (IDEAR), Universidad Católica del Norte, Antofagasta, Chile, e-mail: paroca@ucn.cl

1 Se reconoce y agradece el apoyo del FONDECY, proyecto número 11070168: "Análisis Estructural de la Economía Magallánica Desde la Perspectiva del Enfoque Input-Output” y, del Núcleo de la Iniciativa Científica Milenio "Ciencia Regional y Políticas Públicas". 


\title{
LOST OPPORTUNITIES IN MAGALLANES
}

\begin{abstract}
Many researchers highlight the importance of the relationship between sectors with the objective of suggesting solutions related to economical growing (studies about linkages), but few of them relate such information with the effects of primary inputs and the interregional commutation in the economy. Despite the fact that these types of analysis are different, they are strongly related when we intent to inquire in reasons leading toward to a regional growing.

This work is focused in the analysis of linkages, salaries and regional commutation, considering different sceneries (open and close economy). The result obtained, left clear the lost of resources that are perceived in Magallanes, and have as a destiny other regions, due to the lack of policies that concentrate in establishing the persons that have higher income in the region.
\end{abstract}

KEYWORDS: Input-Output Structural Analysis, Interregional Commutation, Linkage.

JEL Classification

A10, B41, C67, D57, O10, R15

\section{INTRODUCCIÓN}

Un hecho del cual recientemente tenemos información que está ocurriendo en el mercado laboral de las regiones es la conmutación interregional (Aroca y Atienza, 2008). Esta consiste en que los trabajadores han estado separando la decisión de donde vivir de la de donde trabajar. La más plausible explicación de tal acto, está en los diferenciales de costo de vida entre las regiones que son suficientemente grandes como para financiar los gastos de traslados y permitir vivir en aquellas regiones que resultan más atractivas para hacerlo (Paredes y Aroca, 2008).

Según el Censo del año 2002, el 3,7 por ciento de las personas que trabajan en Magallanes, no viven en ella, lo que implica que casi 2000 trabajadores llevan sus ingresos a otras regiones, el 65 por ciento de estos trabajadores, vive, y podemos asumir que gasta sus ingresos, en las regiones Metropolitana, de Valparaíso y O'Higgins. Estos trabajadores, además pertenecen a la parte alta de la distribución de salarios, lo que implica un efecto mayor al 3,7 por ciento, ya que ellos ganan mucho más en remuneraciones que lo representado en igual cantidad de trabajadores con ingresos medios o menores.
Con el objetivo de determinar el impacto que este hecho tiene sobre la región de Magallanes en términos de oportunidades perdidas y, al mismo tiempo, queremos evaluar el efecto positivo que provocan en las regiones de origen los trabajadores que conmutan a esta región. En este trabajo se estima el diferencial que se provoca dado éste hecho en Magallanes, considerando para estos fines, dos escenarios: por un lado, se analiza lo que ocurre asumiendo que los trabajadores de Magallanes gasten sus remuneraciones en la región y, en segundo lugar, se estima en contraposición a lo anterior, el impacto que tiene el que ello no sea así.

Por otro lado, la importancia y extrapolación que se manifiesta al indagar sobre lo expuesto, se puede resumir en los siguientes puntos:

1. La región de Magallanes es una zona extrema, esto es, está aislada geográficamente del resto del país, lo que permite separar ciertos efectos $e$ interrelaciones de mejor manera.

2. La región basa su economía en actividades vinculadas a la extracción del petróleo (commodity).

3. Al ser una zona extrema, se facilita el comprender y cuantificar los efectos que se originan por gastar los salarios en ella versus el que esto último no sea así (economía abierta en contraste a una cerrada). 
4. La región al analizarse como una zona aislada, facilita la implementación de Políticas Públicas que proyecten al arraigo de personas, esto es, se ataca con mayor precisión las causas que hacen que las personas no fijen su residencia en la zona, así como las soluciones a que tal escenario se revierta.

El documento se estructura de la siguiente manera: 1) Se entrega una breve descripción de la economía magallánica desde el año 1960 al 2006; 2) Se analizan y determinan los encadenamientos productivos de la región, considerando dos escenarios (economía abierta y cerrada), esto es, asumiendo primero que se gastan fuera de la región los pagos por los factores productivos e impuestos; y para el segundo caso, se asume que todo se gasta en Magallanes; 3) Se cuantifica el impacto que tiene en la demanda final de la economía magallánica, el hecho que sus trabajadores gasten sus salarios en la región, esto es, asumiendo que trabajan y viven en Magallanes, versus el caso en que trabajen allí pero vivan en otra parte del país; 4) Considerando la información recogida de los CENSOS de 1992 y 2002, se estudian los efectos de la inmigración y conmutación regional, de este análisis se hace patente la necesidad de generar políticas que apunten a lograr que las personas que obtienen mayores salarios vivan en la región; 5) Luego de hacer un cruce entre la información obtenida del CENSO de 2002, de la superintendencia de AFP y la Encuesta CASEN (ambas para el año 2003), se logra establecer cuál es el impacto de la conmutación interregional en el nivel de los salarios para la región de Magallanes; 6) Se hace un análisis de las relaciones de compra y venta de actividades económicas (encadenamientos) y de cómo estas, se distribuyen y afectan el entramado productivo (dispersión) de la región de Magallanes; 7) Finalmente, se presentan algunas conclusiones que dan cuenta del impacto positivo que se produciría en la región si se promueve la radicación en vez de la conmutación a la región de Magallanes.

\section{BREVE DESCRIPCIÓN ECONÓMICA DE MAGALLANES}

Una perspectiva histórica del PIB regional revela la importancia que tuvo en los primeros años para la región la minería. Prácticamente tres cuartos del producto de los años 60 se realizaba en este sector, situación que sin dudas ha cambiado a lo largo del tiempo, mostrando una significativa pérdida en importancia relativa del sector en la distribución actual, como lo muestra la Fig. 1.

De acuerdo a la evolución de la participación sectorial del producto, se observa que a principios de los años ochenta, surgen sectores tales como el de otros servicios y el de industria manufacturera como actividades importantes para la región. En tal sentido, mención especial hacemos de la industria

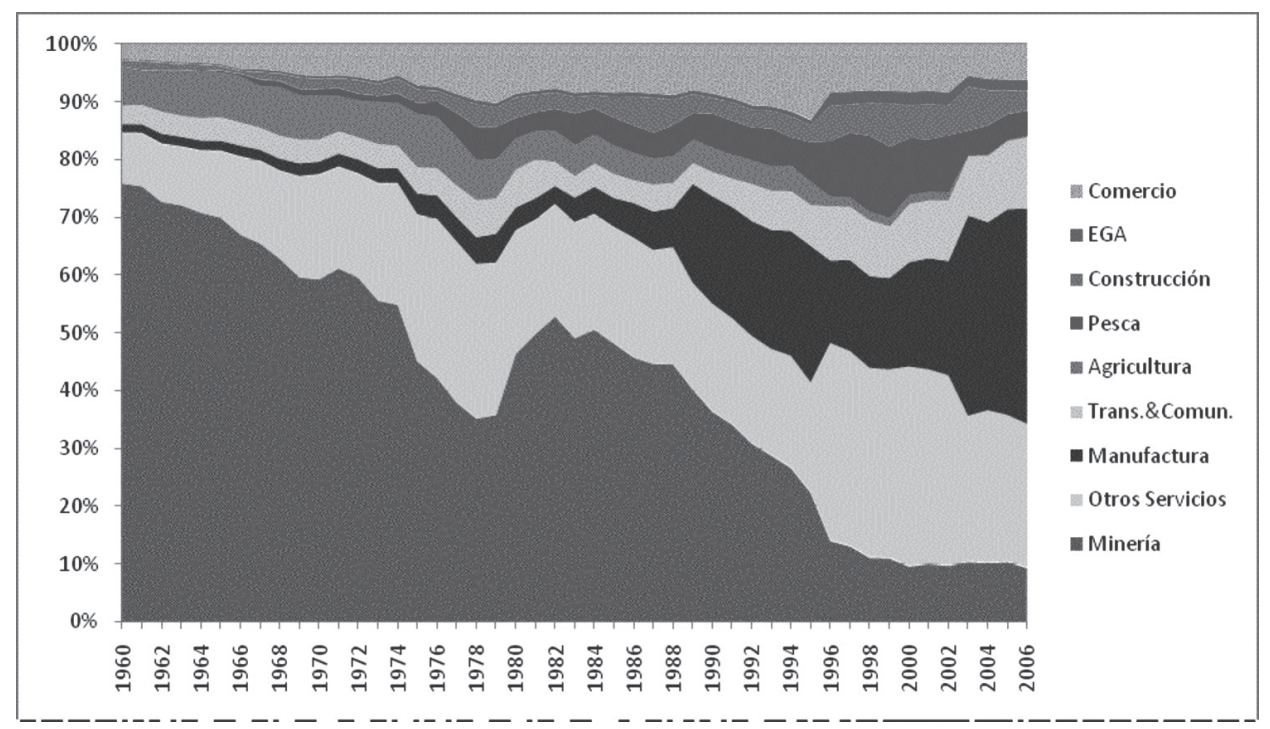

Fig. 1. Participación Sectorial del Producto Geográfico Bruto Regional 1960-2006. Fuente: MIDEPLAN y Banco Central de Chile. 


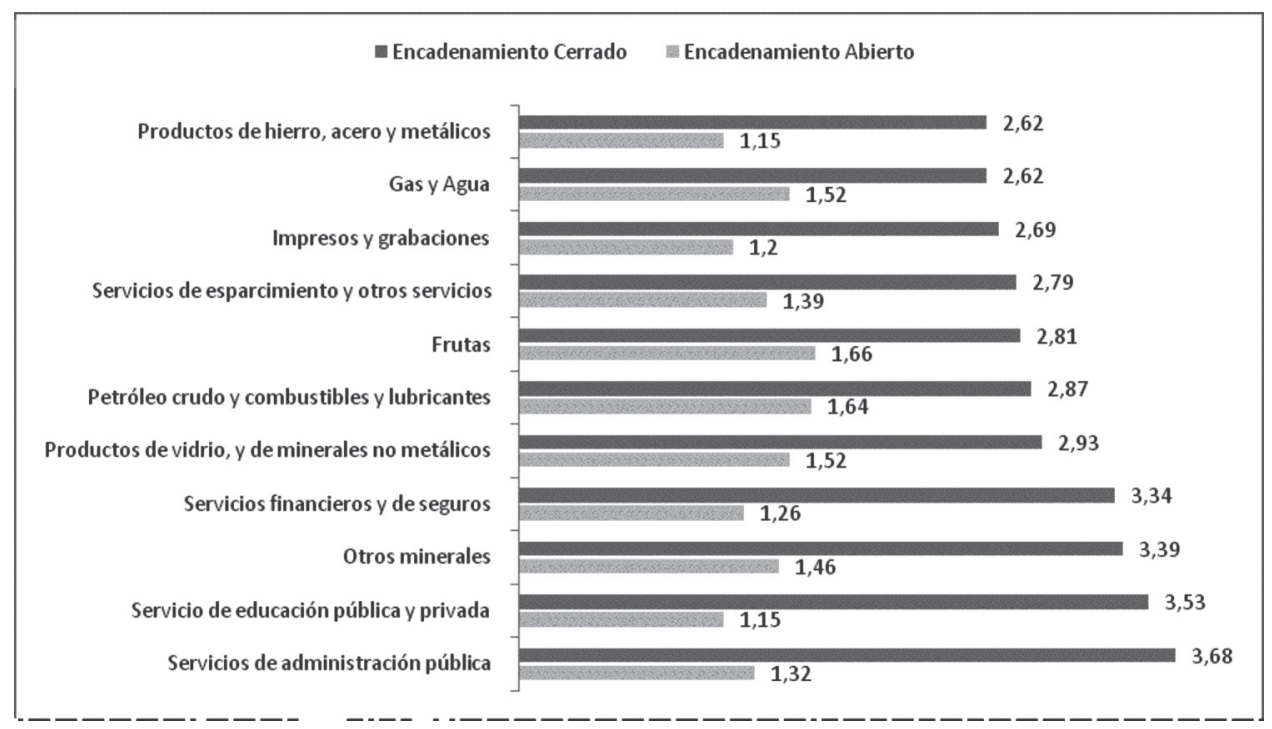

Fig. 2. Mayores Encadenamientos en el Sistema Abierto y Cerrado para Magallanes.

Fuente: Elaboración Propia.

manufacturera, dado que se torna muy relevante después de la crisis asiática; $y$, probablemente y en forma paralela, es la rama otros servicios la que está capturando el impacto del sector turismo que está incorporado dentro del sector comercio, restaurantes y hoteles, el sector transporte y comunicaciones, todos con niveles de participación significativos a través del tiempo.

Por otra parte, la participación del sector agrícola tiende a desaparecer en la región, mientras que la del sector pesca se hace significativa hacia finales de 1990, perdiendo un poco de importancia relativa en la actual década.

Otro elemento que surge del gráfico de participación sectorial es que la economía magallánica pasa desde una altamente concentrada en el sector minero en los sesenta a una economía más diversificada en los últimos años, lo que la hace menos riesgosa o menos vulnerable a los vaivenes internacionales del sector minero. Adicionalmente, muestra una evolución deseable, ya que la diversificación se hace desde el sector primario hacia sectores secundarios y terciarios, lo que es un indicio de un camino hacia el desarrollo.

\section{LOS ENCADENAMIENTOS PRODUCTIVOS}

Los encadenamientos productivos indican cuánto se produce en la región por cada unidad que es consumida, invertida, gastada por el gobierno o exportada al resto del país o al mundo (Hewings 1985, Isard et al, 1998, Miller and Blair, 2009). Al calcular los encadenamientos (multiplicadores) considerando solo las interacciones intersectoriales, se asume implícitamente que los receptores de los pagos de factores productivos (trabajadores y dueños del capital) e impuestos (gobierno) gastan los recursos recibidos fuera de la economía magallánica (esquema conocido como Sistema Abierto).

En un esquema regional, eso puede ser cierto para los dueños del capital y el gobierno, dado que muchos de los primeros no viven en la región y el segundo no gasta en la región de acuerdo a sus niveles de producción sino de acuerdo a otros criterios. Pero los trabajadores, normalmente viven en esas regiones, por lo que asumir que gastan sus salarios en otras regiones es menos plausible. En este sentido, es esperable que los salarios, que reciben los trabajadores, lo gasten en el sistema sectorial local y lo hagan de acuerdo a su estructura de consumo (conocido como Sistema Cerrado). El asumir que los trabajadores gasten sus salarios fuera de la región parece apropiado para aquellos trabajadores que conmutan a la región, es decir, viven en otra región y van a trabajar a la región de Magallanes en un sistema de turno que les permite permanecer en la zona durante los días de trabajo y regresar a su hogar durante los días de descanso (Aroca, 2007). 
La Fig. 2, muestra las 10 actividades económicas, de un total de 40, con mayores encadenamientos para la región de Magallanes (el total de ellas y sus multiplicadores se encuentra en la Tabla 1 del anexo). Dos conclusiones importantes emergen de este gráfico. Primero, la magnitud del impacto de un incremento en la demanda final sobre el producto de la región, se incrementa significativamente al considerar que los salarios se gastan en la región. Segundo, se aprecian variaciones importantes entre los niveles de impacto de los diferentes sectores económicos.

Los multiplicadores que se obtienen cuando la economía está abierta, varían desde 1.2 a 1.66; $y$, cuando se considera cerrada, es decir, cuando todos los salarios, que ganan los trabajadores, se gastan en la región, varían desde 1.65 a 3.68. Lo anterior se debe entender de la siguiente manera: para el primer caso, asúmase a modo de ejemplo, que la actividad "impresos y grabaciones", aumenta su producción en 1 millón de pesos, escenario en el cual la producción total de la economía lo hará en 1.2 veces, es decir, dicho cambio, provocaría un efecto indirecto de 20 por ciento, esto es, de $\$ 200.000$; por otra parte, si dicho incremento se hubiese dado en la agricultura o en los productos frutícolas, el efecto indirecto hubiese sido de un 66\%, es decir, la demanda final se hubiese incrementado, en al menos $\$ 660.000$.
Segundo, es notable el grado de integración que muestra la minería que se desarrolla en la región de Magallanes con la economía local, especialmente a través de los salarios. El petróleo crudo, combustibles y lubricantes, así como otros minerales, están entre los productos que tienen multiplicadores más altos en la economía magallánica. Esto es particularmente relevante porque la minería es una actividad importante en la región y contrasta con el bajo nivel de encadenamientos encontrados para el sector minero de la Región de Antofagasta de acuerdo a Aroca (2001). Los servicios que han crecido significativamente, como lo expone el gráfico 1, también muestran un alto encadenamiento con la economía local, especialmente, a través del gasto de los salarios en la región. También los productos manufacturados están altamente encadenados productivamente con actividades asociados a la minería, mientras que estos son relativamente bajos con el sector de bienes agrícolas, como se puede apreciar en la Fig. 3.

Una actividad, que es importante en Magallanes y que merece atención especial, es el de la industria química, ello dado que la producción del metanol es considerada en este ítem. De acuerdo al sistema abierto, se observa que su multiplicador es de 1.5 , esto quiere decir, que bajo este contexto, por cada millón de pesos que aumenta la demanda final, el sistema lo hace en un 50\%, en otras palabras, el

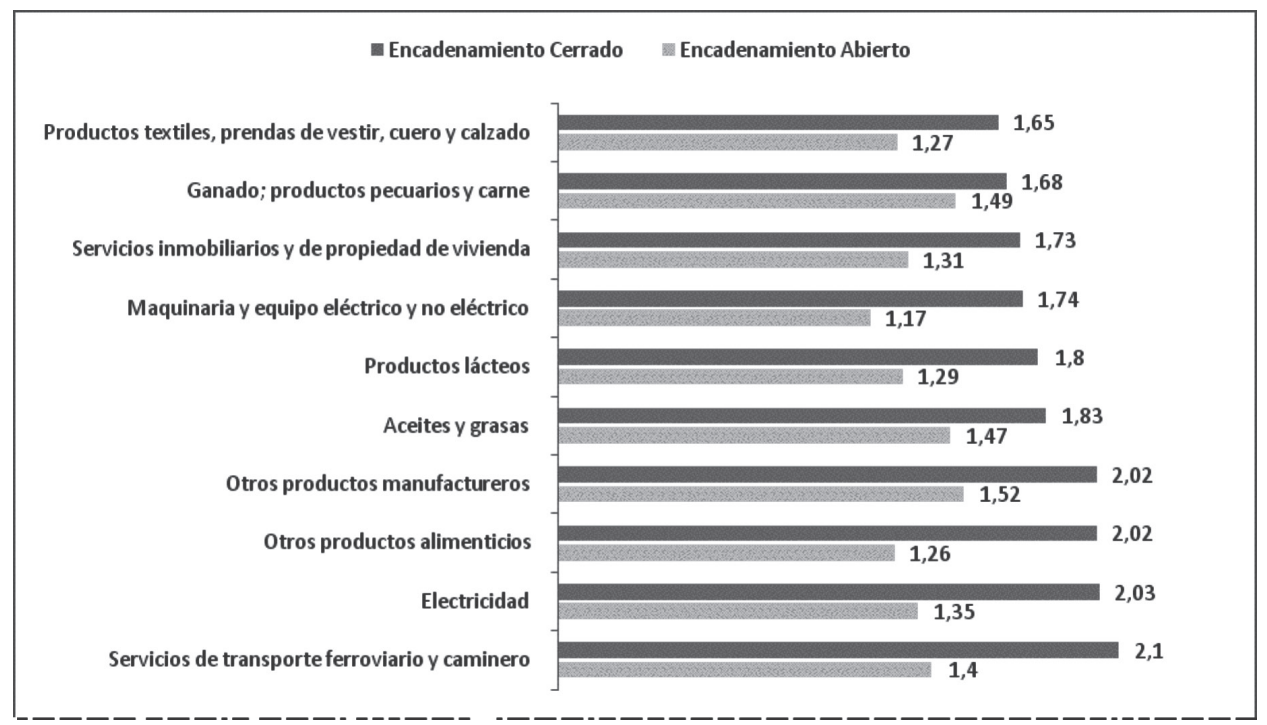

Fig. 3. Menores Encadenamientos Sistema Abierto y Cerrado para Magallanes. Fuente: Elaboración Propia. 
efecto indirecto de tal industria es de 0.50, es decir, $\$ 500.000$ por cada millón demandado.

Cuando se considera a la economía cerrada, se observan importantes diferencias. La principal, es la cuantía de los multiplicadores, ya que en general, cuando el sistema está cerrado, estos alcanzan el doble de los obtenidos asumiendo un sistema abierto. Esto implica que el impacto sobre la producción local, de gastar los salarios en la región duplica el impacto del sistema productivo, este efecto es especialmente significativo en el caso de sectores con alto uso intensivo de la mano de obra.

Lo anterior, es importante, pues retomando el caso de la industria química, se observa que bajo el sistema cerrado, su multiplicador es de 2.15, lo cual implica que si de tal rama se exportasen 1 millón de pesos, la producción adicional indirecta seria de 500 mil pesos, pero además si se considera el efecto inducido de gastar los salarios en la región, habría un incremento de $\$ 650.000$ adicionales. En otras palabras, la economía magallánica, habría incrementado su producción total en 2.15 millones de pesos en total (1.5 más 0.65).

\section{IMPACTO DEL GASTO DE LOS SALARIOS EN LA REGIÓN}

Las Figs. 2 y 3 muestran la importancia del impacto que tienen el gasto de los salarios productos o servicios de la economía regional. Por ejemplo, la Fig. 2 muestra que la actividad otros minerales tendría un impacto sobre el valor bruto de la producción local de 1.46, si los ingresos ganados por los trabajadores asociados a la producción de cada unidad de ella, se gastaran en alguna otra región o país. Mientras que este sube a 3.39 si esos salarios son gastados en la región de Magallanes. Esto da una clara idea de la importancia que tiene el destino de los salarios sobre el impacto en la economía regional.

En este contexto, el hecho que algunos de los trabajadores de la región de Magallanes no gasten los salarios que se paguen por un incremento en la demanda final, dentro de la economía magallánica, implica una pérdida considerable de crecimiento de la región. Una pérdida de oportunidad de desarrollar el sistema productivo local, que es hacia donde está dirigido normalmente el gasto de los salarios de los trabajadores.
La Fig. 2 también muestra que entre las diez actividades con mayor encadenamiento productivo, en siete de ellas, el impacto de gastar los salarios en la región más que duplica el impacto del cambio en la demanda final en algunas de estas. Esto refuerza una lección aprendida desde los estudios de desarrollo económico. No solo es importante crecer, sino que también lo es el generar los mecanismos e instituciones para que ese crecimiento se transforme en crecimiento local y este último, es especialmente potenciado a través del gasto de los salarios en el territorio donde se generan.

La Fig. 3 muestra una situación distinta a la descrita anteriormente. Aquí los encadenamientos productivos son, en general, menores y el impacto que se gasten los salarios en la región aún cuando es considerable, no son tan significativos como los ejemplos vistos previamente, estos son sectores menos encadenados con el sistema productivo local.

Los pagos adicionales a los dueños del capital (excedentes de explotación) y al gobierno (impuestos), es probable que no se gasten en la región, al menos no en proporción del incremento en la demanda.

Al calcular los multiplicadores de productos y encadenamientos productivos a partir de la matriz de Leontief, se aprecia que en promedio, estos aumentan de 1.38 a 1.99 , esto implica que por cada unidad monetaria (millón de pesos) gastado en la economía de Magallanes como demanda final, la economía en cuestión produce en promedio 0.38 unidades monetarias adicionales por las compras al resto de la economía (efecto directo mas indirecto). Por otra parte, los trabajadores de la Región demandarán un conjunto de bienes y servicios usando los salarios recibidos, lo que empujará a las distintas actividades a producir más bienes y servicios para satisfacer esas necesidades. El incremento en producto necesario para satisfacer esas demandas adicionales será de 0.61 unidades monetarias, considerablemente más importante que el generado por el shock de demanda inicial.

La conclusión que se deduce de esta sección es, que no basta con atraer negocios o empresas generadoras de puestos de trabajos a la región, sino que es muy relevante hacerse atractivo en términos de residencia para que los trabajadores decidan vivir en Magallanes y gastar sus salarios en la economía local, esto permitirá desarrollar una economía sustentable en el tiempo. De lo contrario, la economía 
de la región estará en una posición muy vulnerable ante cambios negativos, especialmente en la explotación de recursos naturales.

\section{LA CONMUTACIÓN INTERREGIONAL}

Una de las razones por lo que está cobrando relevancia este hecho, es que existe un proceso creciente de conmutación interregional que ha reemplazado al proceso de migración interregional (Aroca y Atienza, 2008). En el Censo de 1992 la tasa de migración interregional promedio anual era de $1.39 \%$, mientras que en el Censo de 2002, esta cayó a $1.31 \%$, cuando todos los elementos que promueven la migración interregional se movieron en la dirección que hacía prever que ésta aumentaría. Entre estos elementos se cuentan hechos tales como: la baja en los costos de transporte, que los ingresos y la educación promedio subieron, etc. (Aroca y Hewings, 2002 y, Aroca, Hewings y Paredes, 2001). Sin embargo, el Censo de 2002, también nos entregó información por primera vez sobre la conmutación laboral interregional, la cual fue de $2.51 \%$, casi el doble de la tasa de migración de los trabajadores. Esto significa, que se ha hecho mucho más importante la movilidad de trabajadores que van a laborar a una región pero no a vivir en ella. Adicionalmente, esto ocurre para quienes ganan ingresos altos, y para el caso de Magallanes alcanzo a 1.974 trabajadores el 2002. Es decir, personas que trabajaban en la Región de Magallanes pero vivían en otra región y probablemente gastaban la mayor parte de su salario en esa otra región, lo que tenía como consecuencia, que estaban fomentando el crecimiento de la economía de la región donde tenían su residencia a partir de fuentes de trabajo desarrolladas en Magallanes y que implican oportunidades perdidas para esta última.

Esto debe llamar la atención a los planificadores preocupados del crecimiento de Magallanes, es decir, la política no solo debe orientarse a generar fuentes de trabajo, sino también, en hacer más atractiva la región con el fin de que los trabajadores muevan su residencia a esta.

La Fig. 4 muestra que de la mayoría de los casi 2.000 trabajadores que vienen a laborar a Magallanes, lo hacen desde regiones del centro del país. Estas concentran una alta proporción de la economía del país y con este proceso se está ayudando a que esa concentración siga aumentando.

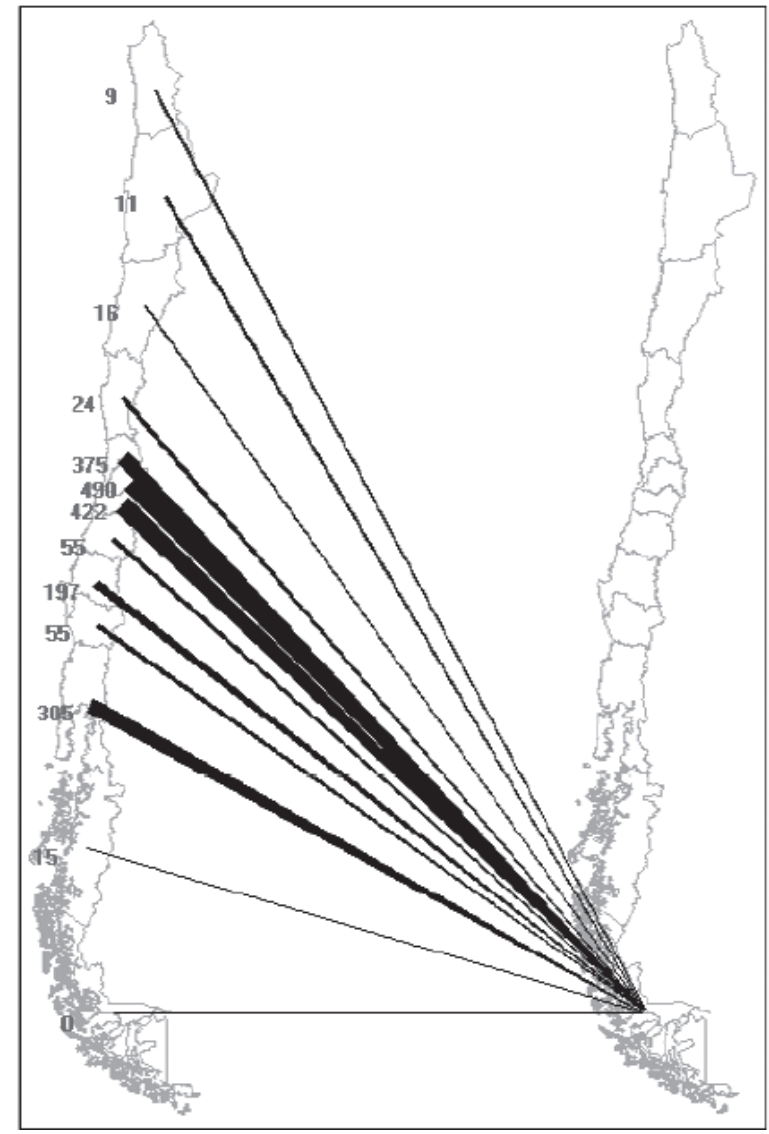

Fig. 4. Conmutantes a la Región de Magallanes Fuente: Elaboración Propia en base a Censo 2002.

\section{IMPACTO DE LA CONMUTACIÓN SOBRE LOS SALARIOS}

Una idea de la magnitud que tiene la conmutación sobre los salarios la da la Fig. 5, donde se muestran los salarios relativos de la Región de Magallanes respecto a los de la Región Metropolitana, obtenidos de dos fuentes, la encuesta CASEN (Encuesta de Caracterización Socioeconómica Nacional) y la Superintendencia de AFPs (Administradoras de Fondos de Pensiones) para el año 2003, se usa este año porque es el más cercano al Censo donde se midió la conmutación, para el cual existe información disponible.

Esta figura muestra que en el año 2003, las empresas que pagaron salarios en la región reportaron un promedio levemente superior al que reportaron las empresas que pagaron salarios en 


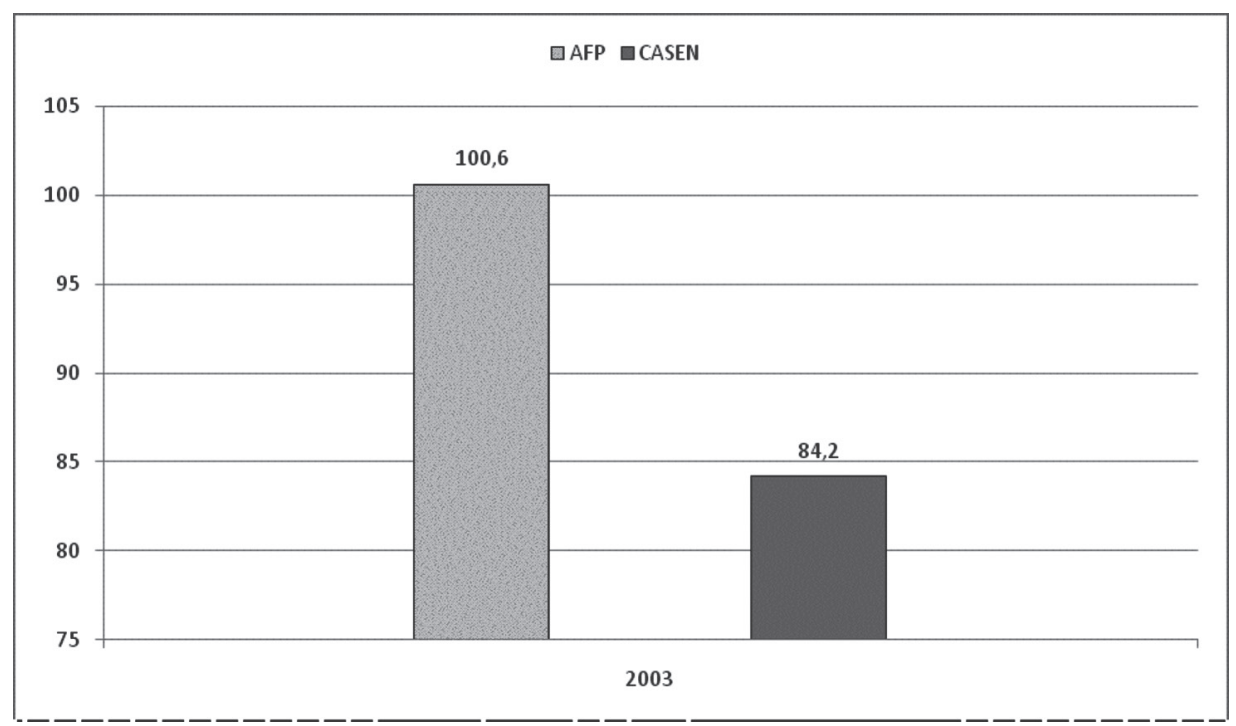

Fig. 5. Salarios Relativos de la Región de Magallanes por Fuentes $(\mathrm{RM}=100)$.

Fuente: Elaboración Propia a Base de Encuesta CASEN y Superintendencia de AFPs.

la Región Metropolitana (0.6\% superior). Por otra parte, la encuesta CASEN, que reporta información sobre la gente que vive en las regiones, muestra que el salario promedio de los trabajadores que vivían en Magallanes en este año, era aproximadamente un $16 \%$ inferior al que recibían los trabajadores de la Región Metropolitana.

Una parte importante de esta diferencia está explicada por la conmutación interregional, las personas que van a trabajar a Magallanes, son aquellas que obtienen salarios altos, de modo que cuando se regresan a sus regiones, el salario promedio que queda para los que viven en la región es considerablemente menor.

Por otra parte, interesante de revisar, es el efecto que se produce en la economía magallánica producto del no gasto de los salarios de las personas que conmutan en la región, esto es, se quiere responder a la pregunta ¿cuál es el impacto que tiene en la economía el hecho de que los que conmutan en Magallanes, no gasten su salario allí?

Del cuadro 1, se aprecia cuál es el efecto de que existan trabajadores, que no residan en la región, asumiendo que $\$ 363.053$ es un salario medio por persona $^{2}$. En la región se dejan de recibir al año

2 Valor muy conservador dado que, son las personas con salarios altos los que conmutan y generan un mayor efecto en los consumos, luego es esperable que sus sueldos e impactos sean mayores. aproximadamente 8.600 millones de pesos ${ }^{3}$, los cuales se distribuyen según la residencia del trabajador, por ejemplo, 1.634 millones de pesos, ganados en Magallanes se gastaron en la región de Valparaíso, estos dineros lo habrían recibido 375 personas que trabajaron en Magallanes, pero fijaron su residencia en aquella región.

De igual modo, se observa que las regiones más beneficiadas con esta fuga de salarios son las de Valparaíso, O’Higgins, Los Lagos y Metropolitana (con, $1.634 ; 1.839 ; 1.329$ y 2.135 millones cada una respectivamente).

\section{ENCADENAMIENTOS DE LA ECONOMÍA MAGALLÁNICA}

Lo que continua, es un análisis que complementa lo anterior, en este sentido, lo que sigue da respuesta a los tipos de actividades que se debe prestar atención a objeto de evitar cambios innecesarios del entramado económico, esto es, se analizan las relaciones de compra y venta que tiene cada acti-

3 Para tener una idea de tal magnitud, puede tomarse como referencia el Total Ejecutado en Inversión Real Efectiva del FNDR (Fondo Nacional de Desarrollo Regional), para el año 2003 en Magallanes, éste fue de MM\$9.371 (ver Gobierno Regional de Magallanes y la Antártica Chilena: Cartera de Proyectos 2003, acezado el 23 de Agosto de 2009. Disponible en: http://164.77.209.180/goremagallanes/archivos/ Cartera2003.htm. 
Cuadro 1. Efecto ingreso de la conmutación en Magallanes (millones de pesos).

\begin{tabular}{|l|r|r|}
\hline Región de origen & $\begin{array}{c}\text { Conmutantes a } \\
\text { Magallanes }\end{array}$ & M\$ \\
\hline Tarapacá & 9 & 39 \\
\hline Antofagasta & 11 & 48 \\
\hline Atacama & 16 & 70 \\
\hline Coquimbo & 24 & 105 \\
\hline Valparaíso & 375 & 1.634 \\
\hline O'Higgins & 422 & 1.839 \\
\hline Maule & 55 & 240 \\
\hline Bio - Bío & 197 & 858 \\
\hline Araucanía & 55 & 240 \\
\hline Los Lagos & 305 & 1.329 \\
\hline Aysén & 15 & 65 \\
\hline Magallanes & 0 & 0 \\
\hline Metropolitana & 490 & 2.135 \\
\hline Total & $\mathbf{1 . 9 7 4}$ & $\mathbf{8 . 6 0 0}$ \\
\hline
\end{tabular}

Fuente: Elaboración propia a partir de tablas insumo- producto de MIDEPLAN, 1996.

vidad, producto o servicio con el resto del sistema económico; así, una actividad será "clave", si tiene una relación de compra y venta -en especial de insumos-, por sobre el promedio; otra será considerada "impulsora", sí, sólo presenta en promedio relaciones de compra; "base", serán aquellas que en términos relativos presenta una alta venta de sus productos pero baja compra de insumos; $y$, finalmente, serán "enclaves" o "isla", aquellas actividades económicas que, vistas en términos relativos, presentan relaciones de compra o venta inferiores o insignificantes.

Para el caso de Magallanes, y siguiendo el enfoque presentado en Soza (2007, ver anexo, Tipo de Multiplicador), se puede observar que existen seis actividades claves: petróleo, combustibles y lubricantes (6), productos químicos, de caucho y de plástico (19), productos de la construcción (27), servicios comerciales (28), servicios de hotelería y de restaurantes (29) y servicios inmobiliarios y propiedad de vivienda (36). Estas además tiene la particularidad de presentar una alta dispersión, tanto en un sentido de oferta como de demanda, esto es, sus implicaciones en términos de efectos distributivos son muy altos, es decir, sus efectos tanto directos como indirectos están altamente dispersos en el sistema económico, lo cual es una característica que promueve la efectividad de políticas públicas orientadas a generar crecimiento. En este sentido, cambios en la demanda de estas actividades altamente interconectadas con el resto del sistema económico, como por ejemplo a través de inversión, afectaran significativamente al resto del sistema productivo. En una línea similar a lo planteado, pero visto desde la vereda contraria, se observa que los productos frutas (2), aceite y grasas (10) y otros productos manufacturados (24), presentan un alto aislamiento respecto al resto del sistema productivo, además, presentan una baja dispersión en términos de compra y venta, exceptuando el producto aceite y grasas, que en compra presenta una baja dispersión, pero alta en el sentido de venta.

En línea con lo expuesto, y revisando la totalidad de las dispersiones y encadenamientos, se observa que para el caso de Magallanes, existe, en general, una alta dispersión y entrelazado entre sus actividades, lo cual muestra lo desarrollado que es ésta economía, en este sentido; $y$, en consonancia con otros trabajos que llegan a similares conclusiones pero utilizando métodos distintos (e.g. Soza en 2008a y 2008b), se puede resaltar dos características; primero, que la estructura de Magallanes es muy profusa; segundo, que al experimentar shock exógenos estos se transmitirán hacia todos los sectores de la economía a través del entramado productivo, especialmente cuando incluya ramas consideradas claves o impulsoras.

Por otra parte, inversiones en aquellos sectores, detectados como "enclaves" - $\mathrm{O}$, en algunos casos, "base"-, con baja dispersión, tendrán efectos directos, como indirectos y distributivos relativamente pequeños. Por lo que una política orientada a promover crecimiento, debería enfocarse en incrementar los encadenamientos en estos sectores, o promover el gasto en los sectores que son claves o impulsores y simultáneamente presentan una alta dispersión de sus efectos.

\section{CONCLUSIONES}

La Región de Magallanes ha evolucionado positivamente en su estructura productiva, pasando de una altamente concentrada en el sector minero a una más diversificada. Sin embargo, esta evolución que caracteriza a los procesos de desarrollo podría no estar siendo aprovechada en toda su magnitud, debido a que una proporción de trabajadores, especialmente aquellos que tienen altos niveles de remuneración, sólo vienen a trabajar a la región y no viven en ella, lo que implica una pérdida de demanda dentro de la economía, con el respectivo desperdicio de todos los efectos positivos que ello implica. 
Los conmutantes vienen principalmente del centro del país, por lo que este proceso de alguna manera refuerza el proceso concentrador de la economía chilena en torno a las regiones del centro y especialmente en torno a la Región Metropolitana.

Una evidencia importante de este efecto negativo se presenta a través de la diferencia entre el nivel de salario promedio que es pagado en la región y el nivel que reciben los trabajadores que viven en Magallanes. Para el año 2003, esta diferencia era de un 16 por ciento. Es decir, las personas que viven en Magallanes ganan en promedio un 16 por ciento menos que el promedio de aquellos que trabajan en esa región. La razón de esta diferencia es que una proporción importante de trabajadores de altos ingresos que laboran en Magallanes viven en otra región del país, especialmente en las regiones del centro. Esto implica que la región del extremo sur de Chile está perdiendo la oportunidad de un mayor desarrollo porque muchos de sus trabajadores no viven en ella y adicionalmente, este efecto es ignorado por el gobierno central a la hora de distribuir sus gastos en el territorio, no considerando el efecto positivo que Magallanes tiene sobre otras regiones.

Finalmente, basados en criterios de encadenamientos y dispersión de los efectos directos e indirectos, se observa que la estructura económica de Magallanes es altamente profusa (entrelazada), esto tiene por ventaja que pequeños shocks económicos en ramas con altos encadenamientos -ramas que demandan y ofertan insumos por sobre el promedio-, y que presentan alta dispersión, generen altos y extensos impactos en el resto de la economía, por lo que promoción, generaría un ahorro considerable de recursos y un incremento significativo en el crecimiento de la economía.

\section{BIBLIOGRAFÍA}

AROCA, PATRICIO. 2007. Migración interna y desarrollo en Chile: Impacto sobre el Crecimiento Regional de la Migración y Conmutación Interregional en Chile (pp. 1-19). Santiago, Chile: CELADE-División de Población. -2001. Impacts and development in local economies based on mining: The case of the Chilean II region. Resources Policy. (27): 119-134.

AROCA, PATRICIO y MIGUEL ATIENZA. 2008. La Conmutación Regional en Chile y su Impacto en la Región de Antofagasta. Revista EURE, XXXIV, (102): 97-120.
AROCA, PATRICIO and GEOFFREY HEWINGS. 2002. Migration and Regional Labor Market Adjustment: Chile 1977-1982 and 1987-1992. The Annals of Regional Science, (36): 197-218

AROCA, PATRICIO and WILLIAMS MALONEY. 2005. Migration, Trade, and Foreign Direct Investment in Mexico. World Bank Economic Review, Oxford University Press, 19(3): 449-472.

AROCA, PATRICIO, GEOFFREY HEWINGS and JIMMY PAREDES. 2001. Migración Interregional y el Mercado Laboral. Cuadernos de Economía, 27(2): 321-345.

HEWINGS, GEOFFREY. 1985. Regional Input-Output Analysis. Beverley Hills: Sage Scientific Geography Series 7, USA.

HEWINGS, GEOFFREY, FONSECA, MANUEL and SONIS, MICHAEL. 1989. Key Sectors and Structural Change in the Brazilian Economy: A comparison of alternative approaches and their policy implications. Journal of Policy Modeling, 11(1):67-90.

HIRSCHMAN, ALBERT. 1958. The Strategy of Economic Development. New Haven, Connecticut, USA, Yale University Press.

ISARD, WALTER, IWAN AZIS, MATTHEW DRENNAN, SIDNEY SALTZMAN and ERIK THORBECKE. 1998. Methods of Interregional and Regional Analysis, Ashgate, Hants, UK.

MIDEPLAN. 2005. Aproximación a las Economías Regionales con base en las Matrices de Insumo Producto Regionales del Año 1996. Santiago, Chile, Ministerio de Planificación. pp. 211.

MILLER, RONALD and PETER BLAIR. 2009. Input-Output Analysis: Foundations and Extensions. Cambridge University Press.

PAREDES, DUSAN y PATRICIO AROCA. 2008. Metodologías Para Estimar un Índice Regional de Costo de Vivienda en Chile. Cuadernos de Economía, 46(134): 129-143.

PULIDO, ANTONIO y FONTELA, EMILIO. 1993. Análisis Input-Output. Modelos Datos y Aplicaciones. España. Editorial Pirámide.

RASMUSSEN, PAUL NOREGAARD. 1956. Studies in Inter -Sectoral Relations. Amterdam, North- Holland P. C.

SOZA-AMIGO, SERGIO. 2008a. Análisis Comparativo para la Economía Magallánica desde la Perspectiva del Enfoque Input-Output. Revista de Análisis Económico, 23(2):79-102. -2008b. Interrelaciones Sectoriales en Magallanes: En busca de su estructura económica. Magallania, 36(1):79102 .

-2007. Análisis Estructural Input-Output: Antiguos problemas y nuevas soluciones. Tesis (Doctorado en Economía Aplicada). Oviedo, España, Universidad de Oviedo, Departamento de Economía Aplicada, 280 p., 2007. 


\section{ANEXOS}

Formulaciones que determinan el tipo de encadenamiento según las distintas técnicas empleadas (hacia atrás $(\mathbf{B L})$ y delante $(\mathbf{F L}))^{4}$.

Ventas ínterindustriales $\left(\mathbf{z}_{\mathbf{i j}}\right)+$ demanda final $\left(\mathbf{c}_{\mathbf{i}} ;\right.$ consumo, ofd; resto de demanda final $)=$ ventas totales $\left(\mathbf{x}_{\mathbf{i}}\right)$ $\sum_{j=1}^{n} z_{i j}+c_{i}+$ ofd $d_{i}=x_{i}$

Compras interindustriales $\left(\mathbf{z}_{\mathbf{i j}}\right)+$ valor agregado $\left(\mathbf{w}_{\mathbf{j}}\right.$ : salarios; $\mathbf{o v}_{\mathbf{j}}$ : resto del valor agregado)+ importaciones $\left(\mathbf{m}_{\mathbf{j}}\right)=$ salidas totales $\left(\mathbf{x}_{\mathbf{j}}\right)$

$\sum_{j=1}^{n} z_{i j}+w_{j}+o v_{j}+m_{j}=x_{j}$

Matriz de Coeficientes Técnicos

$$
A=\left[a_{i j}=\frac{Z_{i j}}{x_{j}}\right]=\left[\begin{array}{cccc}
a_{11} & a_{12} & \cdots & a_{1 n} \\
\vdots & \vdots & \ddots & \vdots \\
a_{n 1} & a_{n 2} & \cdots & a_{n n}
\end{array}\right]
$$

Matriz Inversa de Leontief

$$
(I-A)^{-1}=\left[b_{i j}\right]=\left[\begin{array}{cccc}
b_{11} & b_{12} & \cdots & b_{1 n} \\
\vdots & \vdots & \ddots & \vdots \\
b_{n 1} & b_{n 2} & \cdots & b_{n n}
\end{array}\right]
$$

Multiplicador de Producción

$$
\mathbf{O}_{\mathrm{j}}=\sum_{\mathrm{i}=1}^{\mathbf{n}} \mathbf{b}_{\mathrm{ij}}
$$

Multiplicador de Ingreso

$$
\mathbf{H}_{\mathrm{j}}=\sum_{\mathbf{i}=1}^{\mathbf{n}} \frac{\mathbf{w}_{\mathrm{i}}}{\mathbf{x}_{\mathrm{i}}} \mathbf{b}_{\mathrm{ij}}
$$

Matriz de Coeficientes de Distribución

$$
\overrightarrow{\mathbf{A}}=\left[\overrightarrow{\mathbf{a}}_{\mathrm{ij}}=\frac{\mathbf{Z}_{\mathrm{ij}}}{\mathbf{x}_{\mathrm{i}}}\right]=\left[\begin{array}{cccc}
\overrightarrow{\mathbf{a}}_{11} & \overrightarrow{\mathbf{a}}_{12} & \cdots & \overrightarrow{\mathbf{a}}_{1 \mathrm{n}} \\
\vdots & \vdots & \ddots & \vdots \\
\overrightarrow{\mathbf{a}}_{\mathrm{n} 1} & \overrightarrow{\mathbf{a}}_{\mathrm{n} 2} & \cdots & \overrightarrow{\mathbf{a}}_{\mathrm{nn}}
\end{array}\right]
$$

Matriz Inversa de Ghosh

$$
\overrightarrow{\mathbf{B}}=(\mathbf{I}-\overrightarrow{\mathbf{A}})^{-1}=\left[\overrightarrow{\mathbf{b}}_{\mathbf{i j}}\right]=\left[\begin{array}{cccc}
\overrightarrow{\mathbf{b}}_{11} & \overrightarrow{\mathbf{b}}_{12} & \cdots & \overrightarrow{\mathbf{b}}_{1 \mathbf{n}} \\
\vdots & \vdots & \ddots & \vdots \\
\overrightarrow{\mathbf{b}}_{\mathbf{n} 1} & \overrightarrow{\mathbf{b}}_{\mathrm{n} 2} & \cdots & \overrightarrow{\mathbf{b}}_{\mathbf{n n}}
\end{array}\right]
$$

Soza (2007; descomposición):

$$
\begin{aligned}
& \mathbf{P B L}_{\mathrm{j}}^{\mathrm{c}, \mathrm{p}}=\left(\mathbf{i}^{\mathrm{t}} \mathbf{G}_{22} \mathbf{A}_{21} \mathbf{y}_{1}\right) \mathbf{p}_{\mathrm{j}}
\end{aligned}
$$

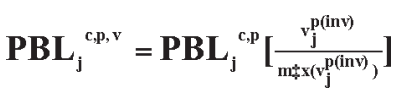

$$
\begin{aligned}
& \text { PFL }_{i}{ }^{\mathrm{G}, \mathrm{p}}=\left(\mathrm{r}_{1} \dot{\mathrm{B}}_{12} \mathrm{~W}_{22} \mathrm{i}^{\mathrm{t}}\right) \mathrm{p}_{\mathrm{i}}
\end{aligned}
$$

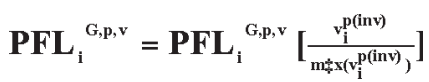


Donde:

n : Corresponde al número de sectores

A : Matriz de coeficientes técnicos, que se descompone en las siguientes submatrices: $\left[\begin{array}{ll}\mathbf{A}_{11} & \mathbf{A}_{12} \\ \mathbf{A}_{21} & \mathbf{A}_{22}\end{array}\right]$

i : vector fila (unitario)

$\mathrm{t} \quad$ : Indica que un vector o matriz está transpuesto

$\check{\mathrm{B}}$ : Matriz inversa de distribución, que se descompone como sigue: $\left[\begin{array}{ll}\overrightarrow{\mathbf{B}}_{11} & \overrightarrow{\mathbf{B}}_{12} \\ \overrightarrow{\mathbf{B}}_{21} & \overrightarrow{\mathbf{B}}_{22}\end{array}\right]$

x : Output (producción total)

y : Demanda final (consumo (privado y del gobierno), formación bruta de capital y exportaciones)

r : Inputs Primarios

w : Salarios

$\mathrm{G}_{22}:\left(\mathrm{I}-\mathrm{A}_{22}\right)^{-1}$

p : Ponderador, $\mathbf{X i} / \mathbf{x}$; para país desarrollado y; Yi/ y; para país en vías de desarrollo

$\mathbf{W}_{22}:\left(\mathbf{i}-\overrightarrow{\mathbf{B}}_{22}\right)^{-1}$

$\mathbf{v}_{j}^{p}$ : Coeficiente de dispersión ponderado $\mathbf{v}_{j}^{\mathbf{p}}=\frac{\sqrt{\frac{\mathbf{1}}{\mathbf{n}-\mathbf{1}} \sum_{\mathrm{i}=1}^{\mathrm{n}}\left(\mathbf{b}_{\mathrm{ij}}-\frac{\mathbf{X}_{\mathrm{j}}}{\mathbf{x}} \mathbf{b}_{\mathrm{j}}\right)^{\mathbf{2}}}}{\frac{\mathbf{X}_{\mathrm{j}}}{\mathbf{x}} \mathbf{b}_{\mathrm{j}}}, \operatorname{con} \mathrm{j}=1,2, \ldots, \mathrm{n} \mathrm{y}, \mathbf{b}_{\mathrm{ij}} \in \mathbf{B}$

$\mathbf{v}_{\mathrm{i}}^{\mathrm{p}}$ : Coeficiente de dispersión ponderado $\quad \mathbf{v}_{\mathrm{i}}^{\mathrm{p}}=\frac{\sqrt{\frac{\mathbf{1}}{\mathbf{n}-\mathbf{1}} \sum_{\mathrm{j}=1}^{\mathrm{n}}\left(\overrightarrow{\mathbf{b}}_{\mathrm{ij}}-\frac{\mathbf{X}_{\mathrm{i}}}{\mathbf{x}} \overrightarrow{\mathbf{b}}_{\mathrm{i}}\right)^{2}}}{\frac{\mathbf{X}_{\mathrm{j}}}{\mathbf{x}} \overrightarrow{\mathbf{b}}_{\mathrm{i}}}$, con i=1, 2, ..., n, y $\overrightarrow{\mathbf{b}}_{\mathrm{ij}} \in \check{\mathrm{B}}$

Tipo de sector según su encadenamiento después de relativizar

$$
\mathrm{BL}>1 \quad \mathrm{BL}<1
$$

FL $>1$

Clave

Base

FL $<1$

Impulsor

Enclave 


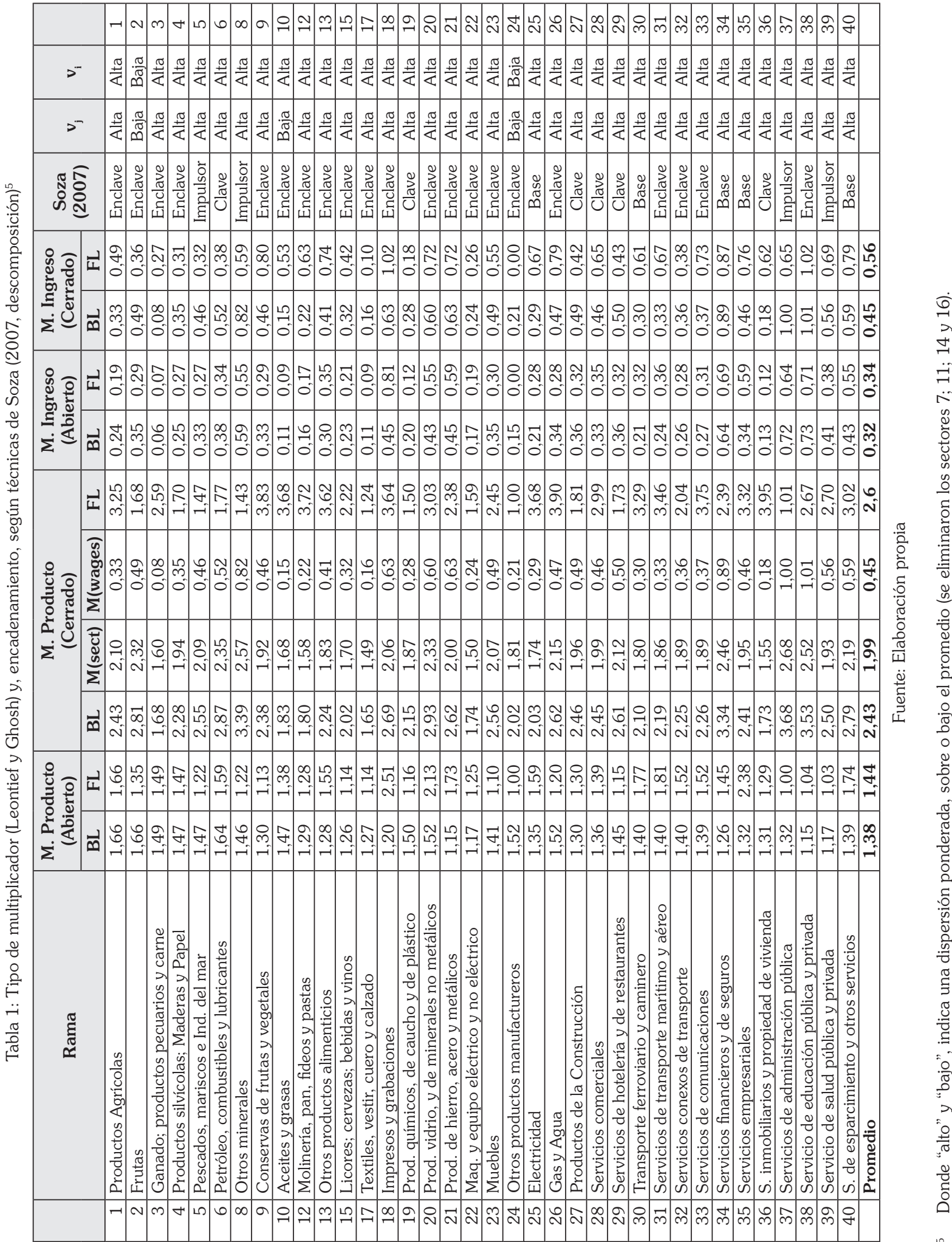


